\title{
BMJ
}

\section{Use of non-steroidal anti-inflammatory drugs and risk of Parkinson's disease: nested case-control study}

\author{
Jane A Driver, assistant professor of medicine, ${ }^{1,2,3}$ Giancarlo Logroscino, associate professor of neurology, ${ }^{4}$ \\ Linda Lu, instructor of medicine, ${ }^{2}$ J Michael Gaziano, professor of medicine, ${ }^{1,2,3,5}$ Tobias Kurth, director of \\ research ${ }^{5,6}$
}

\begin{abstract}
${ }^{1}$ Geriatric Research, Education and Clinical Center, VA Boston Healthcare System; Boston MA, USA

${ }^{2}$ Division of Aging, Department of Medicine, Brigham and Women's Hospital, Harvard Medical School, Boston

${ }^{3}$ Massachusetts Veterans

Epidemiology Research

Information Center, VA Boston

Healthcare System

${ }^{4}$ Department of Neurological and Psychiatric Sciences, University of Bari, Bari, Italy

${ }^{5}$ Division of Preventive Medicine, Brigham and Women's Hospital

${ }^{6}$ INSERM Unit 708-

Neuroepidemiology and UPMC

University Paris 06, Paris, France

Correspondence to: J A Driver,

Division of Aging, Brigham and

Women's Hospital, 1620 Tremont

Street, Boston, MA 02120, USA

jdriver@partners.org
\end{abstract}

Cite this as: BMJ 2011;342:d198 doi:10.1136/bmj.d198

\section{ABSTRACT}

Objective To evaluate the relation between Parkinson's disease and prior use of non-steroidal anti-inflammatory drugs (NSAIDs) in a large cohort of men.

Design Case-control analysis nested in the Physicians' Health Study.

Participants 22007 male physicians aged 40-84 years without indications for or contraindications to regular NSAID use and free of Parkinson's disease at baseline. Cases and controls were matched by age alone or by age and scores for confounders (comorbidity and indicators of NSAID use). Up to five controls were matched to each of 616 cases by age and 565 cases by age and confounder scores.

Setting United States.

Main outcome measures Odds of having been exposed to prior non-aspirin NSAID or aspirin use by participants with Parkinson's disease and by their controls in each casecontrol set.

Results Participants who had ever used non-aspirin NSAIDs had an increased risk of Parkinson's disease (odds ratio $1.28(95 \% \mathrm{Cl} 1.05$ to 1.56$)$ in the age matched group but not in the group also matched on confounder scores (odds ratio 1.17 (0.94 to 1.46)). There was an increased risk of Parkinson's disease in men who had 12 years of regular non-aspirin NSAID use (odds ratio 1.35 (1.07 to 1.70)), a finding that remained significant after matching for confounder scores as well (odds ratio 1.35 (1.05 to 1.75)). In contrast, the significant association of use of non-aspirin NSAIDs for $\geq 5$ years (odds ratio 1.48 (1.05 to 2.09)) in the age matched group was entirely attenuated in the group also matched on confounder scores (1.03 (0.70 to 1.53)). There was also a suggestion that men who regularly used aspirin had an increased risk of Parkinson's disease. Positive associations between non-aspirin NSAID or aspirin and risk of Parkinson's disease tended to disappear when analyses were limited to drug use $\geq 5$ years before the disease diagnosis.

Conclusions This case-control study did not find evidence that NSAID use reduces Parkinson's disease risk. The positive associations observed between NSAID use and Parkinson's disease might have been due to confounding by indication as the use was clustered in the few years before disease diagnosis.

\section{INTRODUCTION}

The evidence that neuroinflammation contributes to the pathogenesis of Parkinson's disease is compelling. ${ }^{1}$ Microglial reaction ${ }^{2}$ and upregulation of inflammatory mediators ${ }^{3}$ are evident in the brains of patients with Parkinson's disease, and a variety of non-steroidal anti-inflammatory drugs (NSAIDS) provide neuroprotection in animal models. ${ }^{4-6}$ Although there is abundant epidemiological evidence of a protective role for NSAIDs in the development of Alzheimer's disease, there have been relatively few observational studies of NSAID use and the risk of Parkinson's disease. Most of these studies have found use of non-aspirin NSAIDs to be associated with a decreased risk of Parkinson's disease, ${ }^{7-15}$ but one reported an increased risk. ${ }^{16}$ Aspirin use has been associated with a decreased risk of Parkinson's disease in three studies, ${ }^{101415}$ and either no effect or increased risk in three others. ${ }^{81217}$ Information on the relation between duration of NSAID use and risk of Parkinson's disease is limited by the relatively short followup of most studies.

Analysis of drug effects in non-randomised studies requires careful consideration for the potential of confounding. There are many chronic conditions associated with NSAID use, and an imbalance in these comorbidities between patients with Parkinson's disease and reference subjects may obscure the association between Parkinson's disease and NSAIDs. Furthermore, Parkinson's disease may cause pain and discomfort years before its diagnosis, ${ }^{18-20}$ creating an association with NSAIDs based on symptoms of the disease itself - a bias known as confounding by indication.

We investigated the relation between use of aspirin and non-aspirin NSAIDs and Parkinson's disease in a large cohort of men with detailed prospective information on drug use and over 25 years of follow-up, with a methodological strategy designed to illustrate the influence of confounding.

\section{METHODS}

The Physicians' Health Study was a randomised trial of aspirin (325 mg every other day) and $\beta$ carotene ( $50 \mathrm{mg}$ 
on alternate days) for the primary prevention of cardiovascular disease and cancer among 22071 US male physicians. Detailed descriptions of the study design and findings have been published. ${ }^{2122}$ At study entry in 1982, participants were between 40 and 84 years; had no history of cardiovascular disease, cancer, or other serious illness; and had no indication for or contraindication to aspirin or non-aspirin NSAIDs. All participants were tolerant to a three month run-in phase of aspirin treatment. Baseline information was self reported and collected by a mailed questionnaire that asked about risk factors for disease as well as lifestyle variables. Ninety two per cent of the participants identified their ethnicity as white. Participants were sent follow-up questionnaires asking about study outcomes and other medical information, twice in the first year and then yearly. The aspirin arm was discontinued after an average of five years of follow-up because of a $44 \%$ risk reduction in myocardial infarction in the aspirin group. ${ }^{21}$ Post-trial follow-up is ongoing. ${ }^{23} \mathrm{We}$ used follow-up information to 4 March 2008 in our analysis.

\section{Ascertainment of Parkinson's disease}

We identified all participants who reported a new diagnosis of Parkinson's disease on their annual survey between 1982 and 2008. To evaluate the accuracy of the self reporting of Parkinson's disease, we performed a validation study using the available medical records of 73 participants who indicated a new diagnosis of Parkinson's disease. ${ }^{24}$ In the Physicians' Health Study, medical records were obtained for each reported study outcome (cardiac event, transient ischaemic attack, stroke, cancer, pulmonary embolism or death). A physician (JAD) evaluated the medical records of participants who reported Parkinson's disease before developing an outcome event. The records were then reviewed independently by two physicians with training in neurology (TK and GL).

The clinical diagnosis of Parkinson's disease was considered valid if record review revealed one or more of

- Established diagnosis of Parkinson's disease in the medical record or Parkinson's disease as cause of death on the death certificate

- Current use of medication for Parkinson's disease such as dihydroxyphenylalanine (DOPA) or a DOPA agonist

- Neurological examination with physical findings consistent with parkinsonism (at least two of rest tremor, rigidity, bradykinesia, and postural instability) with no evidence of a secondary cause of parkinsonism such as stroke, history of encephalitis, brain tumour, or neuroleptic treatment in the year before disease onset. Patients who developed dementia or severe dysautonomia within the first year of diagnosis were not considered valid cases of Parkinson's disease
- Patient with a diagnosis of Parkinson's disease who was followed by a neurologist or a movement disorders specialist.

Of the 73 patients with available medical records, the self reported diagnosis of Parkinson's disease was found to be valid in 90\% (66 patients). In 7\% it was not possible to exclude a secondary cause of parkinsonism, and in only $3 \%$ was the diagnosis of Parkinson's disease incorrect.

\section{Ascertainment of controls}

A participant was considered a control if he was free of self reported Parkinson's disease at the time of the diagnosis of the case (index date) and remained free of the disease for five years from the index date (to avoid the possibility of subclinical disease). However, we considered participants a control if they died within five years of the index date from a cause other than Parkinson's disease to avoid bias. We used information from the questionnaire closest to the index date to define comorbidities and covariates for cases and controls. If this questionnaire was missing, information was imputed from prior questionnaires.

To better understand the influence of comorbidity in our analysis, we created two case-controls sets. In the first set, eligible controls were of the same age as the case. In the second set, participants were matched by age and by scores for confounding variables (comprising a modified Charlson comorbidity score, a score for indicators of NSAID use, and a score for side effects of NSAID use). Controls were selected from the study population in this manner: $(a)$ we matched each case to all potential controls by age at baseline, $(b)$ the date of the case diagnosis of Parkinson's disease was assigned to each potential control as the index date, $(c)$ the comorbidity score and two NSAID scores were calculated for each control at the time of the index date, and (d) up to five matched controls were randomly chosen from all potential controls with the same confounder scores as the case.

\section{Definition of confounder scores}

The comorbidity score was calculated using a modified version of the Charlson comorbidity index, a scoring system for common comorbid conditions that is weighted according to mortality risk. ${ }^{25}$ As participants in the Physicians' Health Study were apparently healthy at baseline, the score reflected total comorbidities accumulated between study entry and the index date. Myocardial infarction, stroke, and cancer were study end points and were confirmed by medical record review. Other conditions were self reported by the study participants. The Charlson index categorises renal and liver disease as mild or as moderate to severe. Because we could not determine disease severity, we categorised all liver disease as mild and all renal disease as moderate to severe, as has been done in other modifications of the Charlson index. ${ }^{26}$ Solid tumours that were localised at presentation received a score of 2 , while those that were metastatic received a score of 6 . 
A score for indications of NSAID use was calculated by assigning one point for each of the following conditions: migraine, frequent headache (reported $\geq 3$ times), osteoarthritis, inflammatory arthritis, cerebrovascular disease, and coronary artery disease. A score for NSAID side effects or contraindications was calculated by assigning a point for each of the following conditions: gastrointestinal bleeding, ulcer, and gastrointestinal symptoms (nausea, vomiting, dyspepsia, dysphagia).

\section{Assessment of NSAID use}

Participants completed annual questionnaires asking about compliance with the study medication (aspirin and $\beta$ carotene), outside use of aspirin, drugs containing aspirin or non-aspirin NSAIDs, and their possible side effects. Non-study aspirin and NSAID use was reported as the number of days of use in the preceding 12 months, and was categorised as $0,1-14,15-60$, or
$>60$ days. Regular use of aspirin and other NSAIDs was defined as $>60$ days' use per year. We included both study and non-study aspirin use in the definition of the aspirin variable. Because few participants had no regular aspirin exposure, cumulative exposure to aspirin was categorised as $0-4,5-9,10-14$, or $\geq 15$ years of regular use. As regular NSAID use was a contraindication to study participation, few participants were regular users of non-aspirin NSAIDs for the first five years of the trial. Cumulative non-aspirin NSAID use was categorised as $0,1-2,3-4$, or $\geq 5$ years.

\section{Statistical analysis}

Using conditional logistic regression, we calculated the odds ratio and 95\% confidence interval of prior non-aspirin NSAID or aspirin use by participants with Parkinson's disease and by their controls in each case-control set. We defined NSAID exposure in three ways: (a) ever versus never regular use for non-aspirin

Table 1|Characteristics and selected comorbidities of Parkinson's disease cases and matched controls among 22007 participants in the Physicians' Health Study. Values are numbers (percentages) of patients unless stated otherwise

\begin{tabular}{|c|c|c|c|c|}
\hline \multirow[b]{2}{*}{ Patient characteristics } & \multicolumn{2}{|c|}{ Matched by age } & \multicolumn{2}{|c|}{ Matched by age and confounder scores* } \\
\hline & Cases $(n=616)$ & Controls $(n=3080)$ & Cases $(n=565)$ & Controls $(n=2458)$ \\
\hline Mean (range) age at randomisation to study (years) & $59.10(40.1-85.0)$ & $59.08(40.0-84.7)$ & $58.57(40.1-85.0)$ & $58.55(40.0-85.0)$ \\
\hline \multicolumn{5}{|l|}{ Mean (range) confounder scores: } \\
\hline Charlson comorbidity score & $1.52(0-11)$ & $1.33(0-10)$ & $1.29(0-7)$ & $1.29(0-7)$ \\
\hline Indications for NSAIDs & $0.92(0-5)$ & $0.84(0-6)$ & $0.83(0-4)$ & $0.83(0-4)$ \\
\hline Side effects from NSAIDs & $0.67(0-3)$ & $0.64(0-3)$ & $0.61(0-3)$ & $0.61(0-3)$ \\
\hline \multicolumn{5}{|l|}{ History of smoking: } \\
\hline Past & 299 (49) & $1550(50)$ & $269(48)$ & $1185(48)$ \\
\hline Current & $15(2.4)$ & $127(4.1)$ & $14(2.5)$ & $102(4.2)$ \\
\hline Takes alcohol daily & $221(36)$ & $1025(33)$ & $200(35)$ & $833(34)$ \\
\hline Rarely takes exercise $†$ & $272(44)$ & $1229(40)$ & $247(44)$ & $921(37)$ \\
\hline \multicolumn{5}{|l|}{ Body mass index: } \\
\hline $25-29$ & $241(39)$ & $1219(40)$ & 220 (39) & $1001(41)$ \\
\hline$\geq 30$ & $39(6.3)$ & $203(6.6)$ & $37(6.6)$ & $151(6.1)$ \\
\hline \multicolumn{5}{|l|}{ History of comorbidities: } \\
\hline Arthritis & $11(1.8)$ & $36(1.2)$ & $6(1.1)$ & $22(0.9)$ \\
\hline Migraine & $73(12)$ & $370(12)$ & $60(11)$ & $237(9.6)$ \\
\hline Chronic headache & $138(22)$ & $690(22)$ & $121(21)$ & $511(21)$ \\
\hline Coronary artery disease & $29(4.7)$ & $127(4.1)$ & $22(3.9)$ & $108(4.4)$ \\
\hline Stroke & $10(1.6)$ & $28(0.9)$ & $7(1.2)$ & $11(0.45)$ \\
\hline Cancer & $102(17)$ & $430(14)$ & $76(14)$ & 291 (12) \\
\hline Diabetes & $8(1.3)$ & $40(1.3)$ & $5(0.9)$ & $16(0.7)$ \\
\hline Peptic ulcer & 115 (19) & $559(18)$ & $97(17)$ & $415(17)$ \\
\hline Gastrointestinal bleeding & $106(17)$ & $464(15)$ & $80(14)$ & $252(10)$ \\
\hline Gastrointestinal symptoms & $25(4.1)$ & $139(4.5)$ & $19(3.4)$ & $85(3.5)$ \\
\hline Congestive heart failure & $36(5.9)$ & $118(3.8)$ & $24(4.3)$ & $59(2.4)$ \\
\hline Pulmonary vascular disease & $40(6.5)$ & $168(5.5)$ & $32(5.7)$ & $107(4.4)$ \\
\hline Liver disease & $82(13)$ & $321(10)$ & $68(12)$ & $250(10)$ \\
\hline Renal disease & $7(1.1)$ & $26(0.8)$ & $4(0.7)$ & $20(0.8)$ \\
\hline Chronic pulmonary disease & $55(8.9)$ & $325(11)$ & $48(8.5)$ & $212(8.6)$ \\
\hline Hemiplegia & $1(0.2)$ & 0 & $1(0.2)$ & 0 \\
\hline Dementia & $30(4.9)$ & $47(1.5)$ & $24(4.3)$ & $19(0.8)$ \\
\hline Randomised to aspirin & 304 (49) & $1532(50)$ & $283(50)$ & $1249(51)$ \\
\hline
\end{tabular}

*Confounder scores=modified Charlson comorbidity score, score for indicators of NSAID use, and score for NSAID side effects.

†Exercise sufficiently vigorous to raise a sweat. 
Table 2|Association between regular use of NSAIDs and risk of Parkinson's disease in cases and matched controls from the Physicians' Health Study. Values are numbers (percentages) of patients unless stated otherwise

\begin{tabular}{|c|c|c|c|c|}
\hline & \multirow[b]{2}{*}{ Cases } & \multirow[b]{2}{*}{ Controls } & \multicolumn{2}{|c|}{ Odds ratio $(95 \% \mathrm{Cl})^{*}$ by drug use } \\
\hline & & & At index date & 5 years prior† \\
\hline Controls matched by age & $n=616$ & $n=3080$ & & \\
\hline \multicolumn{5}{|l|}{ Non-aspirin NSAID use: } \\
\hline Never & $417(68)$ & $2230(72)$ & 1.00 & 1.00 \\
\hline Ever used & $199(32)$ & $850(28)$ & $1.28(1.05$ to 1.56$)$ & 1.18 (0.94 to 1.48$), n=680$ \\
\hline Missing datał & & & & 1.77 (0.59 to 5.27 ), $n=368$ \\
\hline \multicolumn{5}{|l|}{ Aspirin use: } \\
\hline $0-4$ years & $123(20)$ & $680(22)$ & 1.00 & 1.00 \\
\hline$>4$ years & $493(80)$ & $2400(78)$ & $1.20(0.93$ to 1.56$)$ & $1.07(0.84$ to 1.36$)$ \\
\hline $\begin{array}{l}\text { Controls matched by age } \\
\text { and confounder scores§ }\end{array}$ & $n=565$ & $n=2458$ & & \\
\hline \multicolumn{5}{|l|}{ Non-aspirin NSAID use: } \\
\hline Never & $386(68)$ & $1788(73)$ & 1.00 & 1.00 \\
\hline Ever used & $179(32)$ & $670(27)$ & 1.17 (0.94 to 1.46$)$ & 1.02 (0.79 to 1.32$), n=539$ \\
\hline Missing datał & & & & 2.04 (0.53 to 7.78$), n=349$ \\
\hline \multicolumn{5}{|l|}{ Aspirin use: } \\
\hline $0-4$ years & $114(20)$ & $582(24)$ & 1.00 & 1.00 \\
\hline$>4$ years & $451(80)$ & $1876(76)$ & 1.33 (1.00 to 1.78$)$ & 1.01 (0.77 to 1.33$)$ \\
\hline
\end{tabular}

*Estimated by conditional logistic regression. Analyses were adjusted for the following variables at time of matching: smoking (never, past, current), alcohol (daily, weekly, monthly), body mass index $(<25,25-<30, \geq 30$ ), exercise to sweat (ever $v$ never). We adjusted for aspirin use when estimating effects of non-aspirin NSAIDS and vice versa.

†Excluding drug use within 5 years before the index date.

fIndividuals who did not have a variable for NSAID use at 5 years before the index date (they may not have been enrolled in the study).

§Confounder scores=modified Charlson comorbidity score, score for indicators of NSAID use, and score for NSAID side effects.
73.8 years (range 47.5-93.9). The participants with Parkinson's disease were less likely than controls to have smoked and substantially less likely to be current smokers at the time of matching $(2.4 \% v 4.1 \%)$. In the age matched group, cases had higher mean comorbidity scores than controls (1.52 $v 1.33)$. Table 1 shows the characteristics of the cases and controls.

\section{Use of non-aspirin NSAIDs}

In the age matched cohort, those who ever regularly used NSAIDs had a slightly increased risk of Parkinson's disease (odds ratio 1.28 (95\% confidence interval 1.05 to 1.56$)$ ) (see table 2). The odds ratio decreased to $1.17(0.94$ to 1.46$)$ in the group matched for age and confounder scores. When we excluded NSAID use within five years of the matching date, the association in the age matched group disappeared (odds ratio 1.18 (0.94 to 1.48$)$ ).

There was no clear association between NSAID use in the year before diagnosis of Parkinson's disease and risk of Parkinson's disease (table 3). There was an increased risk of Parkinson's disease in men who had regular NSAID use of 1-2 years' duration (odds ratio 1.35 (1.07 to 1.70$)$ ) and of $\geq 5$ years' duration (odds ratio 1.48 (1.05 to 2.09)) in the age matched group, but in the group also matched for confounder scores the risk was increased only in those with 1-2 years of use (odds ratio 1.35 (1.05 to 1.75$)$ ) (table 4). When we excluded NSAID use within five years of the matching date, the association was borderline in the age matched group but no longer apparent in the group also matched for confounder scores. There was no trend towards increased or decreased risk of Parkinson's disease across categories of increasing duration of regular use.

\section{Use of aspirin}

There was a suggestion of an increased risk of Parkinson's disease in men who reported aspirin use for more than four years in the group matched for confounder scores (odds ratio 1.33 (1.00 to 1.78)), but there was no association after excluding aspirin use within five years of the matching date (table 2). There was no association between Parkinson's disease risk and aspirin use in the year before the disease was reported (table 3). Men who reported regular aspirin use of 5-9 years had an increased risk of Parkinson's disease (odds ratio 1.38 (1.02 to1.87)), but the association was not significant for longer durations of use and disappeared after excluding aspirin use within five years of the matching date (table 4).

\section{Tests for effect modification}

There was no significant effect modification by either age or smoking status on the relation between Parkinson's disease and NSAID or aspirin use. Neither a sensitivity analysis excluding those with known vascular disease nor one excluding participants with indicators of chronic pain (headache, migraine, arthritis) changed the results substantially. 


\section{DISCUSSION}

Results from this large nested case-control study do not provide evidence that regular NSAID use decreases the risk of Parkinson's disease. This is despite careful adjustment for comorbid conditions, which can have a substantial influence on the analysis of drug effects in elderly populations. ${ }^{27}$ Non-aspirin NSAID use among cases was clustered in the few years before diagnosis, and positive associations between non-aspirin NSAID or aspirin use and Parkinson's disease tended to disappear with increasing lag time. These findings underscore the complex relation between NSAID use and Parkinson's disease and suggest the presence of confounding by indication.

\section{Comparison with other studies}

A small number of studies provide evidence that NSAID use may decrease risk of Parkinson's disease. In a pooled analysis of data from the Nurses' Health Study and Health Professional Follow-up Study, regular users of non-aspirin NSAIDs at baseline had a relative risk of 0.55 (95\% confidence interval 0.32 to 0.96 ) for Parkinson's disease. ${ }^{10}$ In another large prospective cohort, ibuprofen use was associated with a lower risk of Parkinson's disease (relative risk 0.65 (0.48 to 0.89)), whereas aspirin, acetaminophen, and other NSAIDs had no association. ${ }^{9}$ In one case-control study, a longer duration of regular non-aspirin NSAID use ( $\geq 2$ years) was associated with a substantially decreased risk of Parkinson's disease (odds ratio 0.45 (0.26 to 0.74$)$ ). ${ }^{14}$ Two case-control studies suggest that regular users of aspirin have a lower risk of Parkinson's disease, ${ }^{1415}$ while two others suggest an increased risk. ${ }^{812}$

In contrast, we found no convincing protective association between aspirin or non-aspirin NSAID use and Parkinson's disease. The Rotterdam Study, a population based cohort in which Parkinson's disease was diagnosed by examination in person, also found an increased risk of Parkinson's disease among regular users of NSAIDs in a prospective analysis, although the finding was not statistically significant (relative risk 1.50 (0.95 to 2.37)). ${ }^{16}$ At least three large prospective cohorts found no association between baseline regular use of aspirin and Parkinson's disease risk, ${ }^{910}$ although in two of the cohorts a suggestion of decreased risk was seen in users of higher doses $(\geq 2$ tablets daily). ${ }^{10}$

In some ways the Physicians' Health Study cohort is uniquely suited to investigate the effects of NSAIDs. At baseline, our participants were all tolerant to aspirin and had no imbalance in comorbidities or indications for or against aspirin or NSAID use. Using a matched case-control design, we were able to control for the confounding effect of indications for and contraindications to NSAID use as they developed over time.

However, a number of important distinctions between the Physicians' Health Study and prior cohorts must be considered. Firstly, our population was older than other cohorts, with an average age of 74 years at diagnosis of Parkinson's disease. The effect of a specific exposure can be strongly modified by the
Table $3 \mid$ Association between short term use of NSAIDs and risk of Parkinson's disease in cases and matched controls from the Physicians' Health Study

\begin{tabular}{lcc} 
& \multicolumn{2}{c}{ Odds ratio $(95 \% \mathrm{Cl})^{*}$} \\
\cline { 2 - 3 } Days of use in prioryear & At index date & 5 years priort \\
Controls matched by age & & \\
\hline Non-aspirin NSAID use: & 1.00 & 1.00 \\
\hline None & $0.84(0.65$ to 1.08$)$ & $1.11(0.87$ to 1.43$)$ \\
\hline $1-14$ & $0.87(0.64$ to 1.18$)$ & $1.15(0.86$ to 1.55$)$ \\
\hline $15-60$ & $1.10(0.81$ to 1.50$)$ & $1.09(0.76$ to 1.55$)$ \\
\hline > 60 & 1.00 & 1.00 \\
\hline Aspirin use: & & \\
\hline None & $0.82(0.56$ to 1.18$)$ & $0.96(0.66$ to 1.41$)$ \\
\hline $1-14$ & $1.15(0.76$ to 1.76$)$ & $1.12(0.71$ to 1.78$)$ \\
\hline$>60$ & $0.92(0.74$ to 1.14$)$ & $0.93(0.73$ to 1.18$)$ \\
\hline
\end{tabular}

Controls matched by age and confounder scores $\ddagger$

Non-aspirin NSAID use:

\begin{tabular}{lcc}
\hline None & 1.00 & 1.00 \\
\hline $1-14$ & $0.82(0.63$ to 1.07$)$ & $1.05(0.81$ to 1.37$)$ \\
\hline $15-60$ & $0.71(0.51$ to 0.99$)$ & $1.05(0.76$ to 1.45$)$ \\
\hline$>60$ & $0.82(0.59$ to 1.14$)$ & $0.66(0.44$ to 1.00$)$
\end{tabular}

Aspirin use:

\begin{tabular}{lcc}
\hline None & 1.00 & 1.00 \\
\hline $1-14$ & $0.90(0.60$ to 1.34$)$ & $0.98(0.64$ to 1.48$)$ \\
\hline $15-60$ & $1.29(0.81$ to 2.05$)$ & $1.23(0.75$ to 2.02$)$ \\
\hline$>60$ & $0.99(0.78$ to 1.26$)$ & $0.98(0.75$ to 1.28$)$ \\
\hline
\end{tabular}

*Estimated by conditional logistic regression. Analyses were adjusted for the following variables at time of matching: smoking (never, past, current), alcohol (daily, weekly, monthly), body mass index ( $<25,25-<30$, 230 ), exercise to sweat (ever $v$ never). We adjusted for aspirin use when estimating effects of non-aspirin NSAIDS and vice versa.

†Excluding drug use within 5 years before the index date.

$\ddagger$ Confounder scores=modified Charlson comorbidity score, score for indicators of NSAID use, and score for NSAID side effects.

age at which the cohort begins. In addition, the health conscious physicians in our study are exceptionally long lived, ${ }^{28}$ and $25 \%$ of our cases of Parkinson's disease were diagnosed after age 80 . The protective association of non-aspirin NSAIDs on Parkinson's disease may be stronger in younger patients or in those with younger onset disease. If NSAIDs do delay the onset of Parkinson's disease, this might increase its incidence in old age ${ }^{29}$ However, we had no evidence of effect modification by age in our data. Alternatively, younger patients may have different indications for NSAID use or a longer duration of use than older patients.

Secondly, it is possible that, because of the highly selected nature of our cohort, we are missing a group in which a protective benefit of NSAIDs might be seen. However, there is no plausible reason to believe that the effect of NSAIDs on Parkinson's disease differs in the participants of the Physicians' Health Study as compared with other populations.

Finally, we were unable to separate the exposure to non-aspirin NSAIDs into individual NSAID types, such as ibuprofen (which may be particularly protective against Parkinson's disease).${ }^{9}$ However, at least in early years of the study, ibuprofen was the most commonly used non-aspirin NSAID on the US market. ${ }^{30}$ 
Table $4 \mid$ Association between cumulative regular use of NSAIDs and risk of Parkinson's disease in cases and matched controls from the Physicians' Health Study. Values are numbers (percentages) of patients unless stated otherwise

\begin{tabular}{|c|c|c|c|c|}
\hline \multirow[b]{2}{*}{ Years of regular drug use } & \multirow[b]{2}{*}{ Cases } & \multirow[b]{2}{*}{ Controls } & \multicolumn{2}{|c|}{ Odds ratio $(95 \% \mathrm{Cl})^{\star}$ by drug use } \\
\hline & & & At index date & 5 years priort \\
\hline Controls matched by age & $\mathrm{n}=616$ & $\mathrm{n}=\mathbf{3 0 8 0}$ & & \\
\hline \multicolumn{5}{|l|}{ Non-aspirin NSAID use: } \\
\hline 0 & $417(68)$ & $2232(72)$ & 1.00 & 1.00 \\
\hline $1-2$ & $121(20)$ & $492(16)$ & 1.35 (1.07 to 1.70$)$ & 1.30 (0.99 to 1.69$)$ \\
\hline $3-4$ & $29(4.7)$ & $174(5.7)$ & 0.91 (0.60 to 1.37 ) & 0.74 (0.45 to 1.23$)$ \\
\hline$\geq 5$ & $49(8.0)$ & $182(5.9)$ & $1.48(1.05$ to 2.09$)$ & 1.31 (0.82 to 2.09$)$ \\
\hline \multicolumn{5}{|l|}{ Aspirin use: } \\
\hline $0-4$ & $124(20.1)$ & $693(22.5)$ & 1.00 & 1.00 \\
\hline $5-9$ & $173(28.1)$ & $836(27.2)$ & $1.22(0.92$ to 1.62$)$ & 1.05 (0.80 to 1.36$)$ \\
\hline $10-14$ & $154(25.0)$ & $730(23.7)$ & $1.26(0.93$ to 1.72$)$ & 1.28 (0.95 to 1.73$)$ \\
\hline$\geq 15$ & $165(26.8)$ & $821(26.7)$ & 1.21 (0.88 to 1.68$)$ & 1.05 (0.72 to 1.52$)$ \\
\hline $\begin{array}{l}\text { Controls matched by age } \\
\text { and confounder scores } \ddagger\end{array}$ & $n=565$ & $\mathrm{n}=2458$ & & \\
\hline \multicolumn{5}{|l|}{ Non-aspirin NSAID use: } \\
\hline 0 & $386(68)$ & $1789(73)$ & 1.00 & 1.00 \\
\hline $1-2$ & $111(20)$ & 370 (15) & 1.35 (1.05 to 1.75$)$ & 1.12 (0.84 to 1.50$)$ \\
\hline $3-4$ & $27(4.8)$ & $137(5.6)$ & $0.82(0.52$ to 1.29$)$ & 0.75 (0.42 to 1.36$)$ \\
\hline$\geq 5$ & $41(7.3)$ & $162(6.6)$ & $1.03(0.70$ to 1.53$)$ & 0.81 (0.46 to 1.43$)$ \\
\hline \multicolumn{5}{|l|}{ Aspirin use: } \\
\hline $0-4$ & $115(20.35)$ & $591(24.0)$ & 1.00 & 1.00 \\
\hline $5-9$ & $165(29.2)$ & $656(26.7)$ & 1.38 (1.02 to 1.87 ) & 0.96 (0.72 to 1.28$)$ \\
\hline $10-14$ & $138(24.4)$ & $579(23.6)$ & 1.30 (0.93 to 1.84$)$ & 1.29 (0.92 to 1.80$)$ \\
\hline$\geq 15$ & $147(26.0)$ & $632(25.7)$ & $1.31(0.92$ to 1.86$)$ & 1.02 (0.68 to 1.52$)$ \\
\hline
\end{tabular}

*Estimated by conditional logistic regression. Analyses were adjusted for the following variables at time of matching: smoking (never, past, current), alcohol (daily, weekly, monthly), body mass index $(225,25-<30, \geq 30)$ exercise to sweat (ever $v$ never). We adjusted for aspirin use when estimating effects of non-aspirin NSAIDS and vice versa.

†Excluding drug use within 5 years before the index date. In these analyses, we controlled for missing observations using an indicator variable. For non-aspirin NSAID use, there were 369 individuals missing

information in the age matched group and 349 in the group matched by age and confounder scores. For aspirin use, there were 368 missing in the age matched group and 349 in the group matched by age and confounder scores.

‡Confounder scores=modified Charlson comorbidity score, score for indicators of NSAID use, and score for NSAID side effects. aspirin use in cases was clustered in the few years before the diagnosis of Parkinson's disease.

Pain is common in Parkinson's disease and may be a primary or secondary symptom. ${ }^{32}$ Primary sensory symptoms originate in the peripheral or central nervous system, while secondary pain may be caused by conditions such as dystonia and muscle cramps. Sensory symptoms may precede the diagnosis of Parkinson's disease for several years, even before motor symptoms develop. ${ }^{1920}$ As we did not have the date of symptom onset, it is possible that our patients with Parkinson's disease experienced increased pain for a few years before the diagnosis, explaining the increase in NSAID use around this time.

\section{Strengths and limitations of study}

Our analysis has a number of strengths. Valid data on NSAID use is difficult to obtain through retrospective means or pharmacy databases, since many NSAIDs may be obtained over the counter and are often used periodically. Unlike other studies, we present data on aspirin and non-aspirin NSAID use collected yearly over 25 years. The physicians who participated in this study (a trial of aspirin for prevention of cardiovascular disease and cancer) were particularly motivated to report NSAID use accurately. Participants in the Physicians' Health Study had no indication or contraindication for NSAID use at study entry. As we also have detailed information about comorbid conditions during follow-up, our study is particularly well suited to address concerns about indication bias. Since profession and socioeconomic status are known to be associated with the risk of Parkinson's disease, ${ }^{33}$ the fact that all our participants were physicians should help to reduce confounding. The long follow-up allowed us to examine the effects of duration of treatment, an advantage over other studies.

A particular strength of our study is the nested casecontrol design, which allowed for matching on comorbidities and indicators of chronic NSAID use. This enabled us to evaluate potential confounding effects in a more careful way than is possible in a cohort design. In addition, this design also takes into account potential effect modification by the matching factors on the association between NSAID use and Parkinson's disease, a fact often ignored in multivariable analysis of cohort studies. ${ }^{34}$ To be sure that 1:5 matching was adequate, we performed a bootstrap analysis which provided similar results, reassuring us that our findings are robust.

Our study also has limitations. Firstly, our diagnosis of Parkinson's disease was based on self reporting by the participating physicians. However, the self reported diagnosis of Parkinson's disease is known to be highly valid in a population of health professionals. ${ }^{17}$ In our study, direct validation of more than $10 \%$ of the self reported cases of Parkinson's disease diagnoses by means of medical records revealed an accuracy of $90 \%$, which is similar to that found in other validation studies of self reporting in the Physicians' Health Study. ${ }^{35}$ As we did not have the date matched group and between cumulative aspirin use and Parkinson's disease in the group also matched for confounders. These associations tended to disappear with increasing lag time, suggesting that NSAID and 


\section{WHAT IS ALREADY KNOWN ON THIS SUBJECT}

Inflammation plays an important role in the pathogenesis of Parkinson's disease, and nonsteroidal anti-inflammatory drugs (NSAIDs) may have neuroprotective features. Few population based studies have investigated the relation between NSAID use and Parkinson's disease, and available results are conflicting

\section{WHAT THIS STUDY ADDS}

This nested case-control study among initially healthy male physicians found no evidence that regular use of either non-aspirin NSAIDs or aspirin reduces the risk of Parkinson's disease

The positive associations observed between NSAID use and Parkinson's disease might have been due to confounding by indication

of onset of Parkinson's symptoms, participants may have had the disease for months to years before reporting it. To address this, we excluded data for the five years before the date of reporting the Parkinson's disease diagnosis. Our cohort is homogeneous in a number of important respects, being composed of men of the same educational level and profession who are predominantly white. Hence, generalisability to other populations may be limited. The median age of onset of Parkinson's disease in our cohort is older than in many other studies, and our results may not be applicable to people with early onset disease. However, early onset Parkinson's disease affects only a small portion of patients. We performed a large amount of subgroup analyses, which may increase the risk of chance findings. Finally, despite our attempts to adjust for confounding, our results may be limited by the presence of residual and unmeasurable confounding in this observational study.

\section{Implication for clinical practice}

NSAIDs have been proposed as potential preventive agents for Parkinson's disease, based on laboratory evidence as well as results from a few large observational studies. ${ }^{36}$ However, a number of subsequent studies, including ours, suggest no protective effect. Considering the substantial potential side effects of chronic NSAID use, the totality of available data is not sufficient to recommend NSAIDs for prevention of Parkinson's disease.

\section{Direction for future research}

Because of the inconsistencies in studies evaluating NSAIDs as neuroprotective agents, further studies are warranted to prove or disprove this hypothesis. Such studies should take standard indication, contraindication, and side effects of NSAIDs into account and should carefully evaluate the timing between NSAID use and disease onset.

In summary, in this prospective case-control study of US male physicians, we found no evidence of a protective association between regular use of aspirin or other NSAIDs and the risk of Parkinson's disease. Our results show complex associations, and the positive associations we observed between NSAIDs and Parkinson's disease may be due to confounding by indication, as the NSAID use was clustered in the few years before diagnosis.

We thank the staff of the Physician's Health Study and the 22071 dedicated physicians who have made this project possible.

Contributors: JAD conceived and designed the study, performed the data analyses, wrote the first draft of the article, and is guarantor for the study. $\mathrm{LL}$ helped with the research and drafting of the manuscript. JMG, GL, and TK gave detailed advice at all stages of the analyses. All authors contributed to the writing of the paper and gave substantial advice and input into the study. All authors had full access to the data (including statistical reports and tables) in the study and can take responsibility for the integrity of the data and the accuracy of the data analysis. Funding: This research is funded by a grant from the Parkinson's Disease Foundation. The Physicians' Health Study is supported by grants CA34944, CA-40360, and CA-097193 from the National Cancer Institute and grants HL-26490 and HL-34595 from the National Heart, Lung, and Blood Institute, Bethesda, MD. The study sponsor had no role in study design; data collection, analysis, or interpretation; writing the article; or the decision to submit it for publication. The researchers are independent from funders and sponsors.

Competing interests: All authors have completed the Unified Competing Interest form at www.icmje.org/coi_disclosure.pdf (available on request from the corresponding author) and declare: JAD has support from the Parkinson's Disease Foundation for the submitted work; no financial relationships with any organisations that might have an interest in the submitted work in the previous three years; and no other relationships or activities that could appear to have influenced the submitted work. We report a full disclosure for the past five years for each of the authors JAD has a research grant from the Parkinson's Disease Foundation, the Hartford Foundation, Harvard Medical School, and a Career Development Award from the Veteran's Administration. LL has nothing to disclose. GL has received research funding from the Amyotrophic Lateral Sclerosis Association, Italian Ministry of Health, the Apulia Region, and special funding from the Italian Ministry of Universities and has received honorariums from Pfizer, Novartis, Glaxo, and Lilly Pharmaceutical for speaking engagements. JMG has received investigator initiated research funding and support as principal investigator from National Institutes of Health, BASF, DSM Pharmaceuticals, Wyeth Pharmaceuticals, McNeil Consumer Products, and Pliva; received honoraria from Bayer and Pfizer for speaking engagements; and is a consultant for Bayer, McNeil Consumer Products, Wyeth Pharmaceuticals, Merck, Nutraquest, and GlaxoSmithKline. TK has received investigator initiated research funding from the French National Research Agency, the US National Institutes of Health, Merck, the Migraine Research Foundation, and the Parkinson's Research Foundation; is a consultant to i3 Drug Safety and World Health Information Science Consultants, LLC; and has received honoraria from the American Academy of Neurology, Genzyme, Merck, and Pfizer for educational lectures.

Ethical approval: All participants in the Physician's Health Study provided written informed consent, and the trial was approved by the institutional review board of the Brigham and Women's Hospital. This study, which used analysis of existing data, was also approved by the institutional review board of the Brigham and Women's Hospital.

Data sharing: No additional data available.

1 Hunot S, Hirsch EC. Neuroinflammatory processes in Parkinson's disease. Ann Neurol 2003;53(suppl 3):S49-60.

2 McGeer PL, Schwab C, Parent A, Doudet D. Presence of reactive microglia in monkey substantia nigra years after 1-methyl-4-phenyl1,2,3,6-tetrahydropyridine administration. Ann Neurol 2003;54:599-604.

3 Nagatsu T, Mogi M, Ichinose $\mathrm{H}$, Togari A. Changes in cytokines and neurotrophins in Parkinson's disease. J Neural Transm Suppl 2000;(60):277-90.

4 Aubin N, Curet O, Deffois A, Carter C. Aspirin and salicylate protect against MPTP-induced dopamine depletion in mice. J Neurochem 1998;71:1635-42.

5 Mohanakumar KP, Muralikrishnan D, Thomas B. Neuroprotection by sodium salicylate against 1-methyl-4-phenyl-1,2,3,6tetrahydropyridine-induced neurotoxicity. Brain Res 2000;864:281-90.

6 Teismann P, Ferger B. Inhibition of the cyclooxygenase isoenzymes COX-1 and COX-2 provide neuroprotection in the MPTP-mouse model of Parkinson's disease. Synapse 2001;39:167-74.

7 Administration on Aging. A profile of older Americans: 2005. US Department of Health and Human Services, 
2005.www.aoa.gov/AoAroot/Aging_Statistics/Profile/2005/ 2005profile.pdf

8 Bower JH, Maraganore DM, Peterson BJ, Ahlskog JE, Rocca WA. Immunologic diseases, anti-inflammatory drugs, and Parkinson disease: a case-control study. Neurology 2006;67:494-6.

9 Chen H, Jacobs E, Schwarzschild MA, McCullough ML, Calle EE, Thun MJ, et al. Nonsteroidal antiinflammatory drug use and the risk for Parkinson's disease. Ann Neurol 2005;58:963-7.

10 Chen H, Zhang SM, Hernan MA, Schwarzschild MA, Willett WC, Colditz GA, et al. Nonsteroidal anti-inflammatory drugs and the risk of Parkinson disease. Arch Neurol 2003;60:1059-64.

11 Hancock DB, Martin ER, Stajich JM, Jewett R, Stacy MA, Scott BL, et al. Smoking, caffeine, and nonsteroidal anti-inflammatory drugs in families with Parkinson disease. Arch Neurol 2007;64:576-80.

12 Hernan MA, Logroscino G, Garcia Rodriguez LA. Nonsteroidal antiinflammatory drugs and the incidence of Parkinson disease. Neurology 2006;66:1097-9.

13 Powers KM, Kay DM, Factor SA, Zabetian CP, Higgins DS, Samii A, et al. Combined effects of smoking, coffee, and NSAIDs on Parkinson's disease risk. Mov Disord 2008;23:88-95.

14 Ton TG, Heckbert SR, Longstreth WT Jr, Rossing MA, Kukull WA, Franklin GM, et al. Nonsteroidal anti-inflammatory drugs and risk of Parkinson's disease. Mov Disord 2006;21:964-9.

15 Wahner AD, Bronstein JM, Bordelon YM, Ritz B. Nonsteroidal antiinflammatory drugs may protect against Parkinson disease. Neurology 2007;69:1836-42.

16 Bornebroek M, de Lau LM, Haag MD, Koudstaal PJ, Hofman A, Stricker $\mathrm{BH}$, et al. Nonsteroidal anti-inflammatory drugs and the risk of Parkinson disease. Neuroepidemiology 2007;28:193-6.

17 Chen H, Zhang SM, Schwarzschild MA, Hernan MA, Ascherio A. Physical activity and the risk of Parkinson disease. Neurology 2005;64:664-9.

18 Ford B. Parkinson disease: pain in Parkinson disease: the hidden epidemic. Nat Rev Neurol 2009;5:242-3.

19 Koller WC. Sensory symptoms in Parkinson's disease. Neurology 1984;34:957-9.

20 Sandyk R. Back pain as an early symptom of Parkinson's disease. $S$ Afr Med J 1982;61:3.

21 Steering Committee of the Physicians' Health Study Research Group. Final report on the aspirin component of the ongoing Physicians' Health Study. N Engl J Med 1989;321:129-35.

22 Hennekens CH, Buring JE, Manson JE, Stampfer M, Rosner B, Cook NR, et al. Lack of effect of long-term supplementation with beta carotene on the incidence of malignant neoplasms and cardiovascular disease. N Engl J Med 1996;334:1145-9.

23 Christen WG, Gaziano JM, Hennekens CH. Design of Physicians' Health Study II-a randomized trial of beta-carotene, vitamins $E$ and
C, and multivitamins, in prevention of cancer, cardiovascular disease, and eye disease, and review of results of completed trials. Ann Epidemiol 2000;10:125-34.

24 Driver JA, Kurth T, Buring JE, Gaziano JM, Logroscino G. Prospective case-control study of nonfatal cancer preceding the diagnosis of parkinson's disease. Cancer Causes Control 2007;18:705-11.

25 Charlson ME, Pompei P, Ales KL, MacKenzie CR. A new method of classifying prognostic comorbidity in longitudinal studies: development and validation. J Chronic Dis 1987;40:373-83.

26 Goldstein LB, Samsa GP, Matchar DB, Horner RD. Charlson Index comorbidity adjustment for ischemic stroke outcome studies. Stroke 2004;35:1941-5.

27 Asmis TR, Ding K, Seymour L, Shepherd FA, Leighl NB, Winton TL, et al. Age and comorbidity as independent prognostic factors in the treatment of non small-cell lung cancer: a review of National Cancer Institute of Canada Clinical Trials Group trials. J Clin Oncol 2008;26:54-9.

28 Yates LB, Djousse L, Kurth T, Buring JE, Gaziano JM. Exceptional longevity in men: modifiable factors associated with survival and function to age 90 years. Arch Intern Med 2008;168:284-90.

29 Breitner JC, Haneuse SJ, Walker R, Dublin S, Crane PK, Gray SL, et al. Risk of dementia and AD with prior exposure to NSAIDs in an elderly community-based cohort. Neurology 2009;72:1899-905.

30 Royer GL, Seckman CE, Welshman IR. Safety profile: fifteen years of clinical experience with ibuprofen. Am J Med 1984;77:25-34.

31 Driver JA, Kurth T, Buring JE, Gaziano JM, Logroscino G. Parkinson disease and risk of mortality: a prospective comorbidity-matched cohort study. Neurology 2008;70:1423-30.

32 Snider SR, Sandyk R. Sensory dysfunction. In: Koller WC, ed. Handbook of Parkinson's disease. Marcel Dekker, 1987:171-80.

33 Frigerio R, Elbaz A, Sanft KR, Peterson BJ, Bower JH, Ahlskog JE, et al. Education and occupations preceding Parkinson disease: a population-based case-control study. Neurology 2005;65:1575-83.

34 Kurth T, Walker AM, Glynn RJ, Chan KA, Gaziano JM, Berger K, et al. Results of multivariable logistic regression, propensity matching, propensity adjustment, and propensity-based weighting under conditions of nonuniform effect. Am J Epidemiol 2006;163:262-70.

35 Camargo CA Jr, Hennekens CH, Gaziano JM, Glynn RJ, Manson JE, Stampfer MJ. Prospective study of moderate alcohol consumption and mortality in US male physicians. Arch Intern Med 1997;157:79-85.

36 Schiess $M$. Nonsteroidal anti-inflammatory drugs protect against Parkinson neurodegeneration: can an NSAID a day keep Parkinson disease away? Arch Neurol 2003;60:1043-4.

Accepted: 19 October 2010 